\title{
ZAPATERO A TUS ZAPATOS. APRENDIZAJE DE OFICIOS
}

\section{LET TO THE COBBLER, STICK TO HIS LAST. LEARNING OF TRADES}

\section{Agustina María Manavella ${ }^{a}$, Rocio Belén Martín $^{b}$ y Danilo Silvio Donolo}

Fechas de recepción y aceptación: 26 de marzo de 2019, 4 de septiembre de 2019

DOI: 10.46583/edetania_2020.57.443

Resumen: El escrito apunta a avanzar en el estudio del aprendizaje de oficios, en diversos contextos -no formales e informales-; reconociendo la importancia que adquieren ciertos aspectos personales y contextuales en los aprendizajes para el trabajo. Se realizó un estudio de caso múltiple, considerándose dos casos: el caso Clavito, tallerista de una capacitación en reparación de calzado, quien aprendió su oficio en contextos informales, y el caso Álvaro, participante de la capacitación. Los resultados obtenidos permiten reconocer la interacción entre aspectos personales y contextuales, que participan y resultan promisorios para el aprendizaje de oficios, permitiendo repensar estos contextos y la importancia de continuar trabajando en el diseño de estrategias y formas de enseñanza en los espacios de capacitación laboral.

Palabras clave: aprendizaje, oficios, contextos no formales, aprendizaje informal.

${ }^{a}$ Instituto Académico Pedagógico de Ciencias Sociales. Universidad Nacional de Villa María. Consejo Nacional de Investigaciones Científicas y Técnicas.

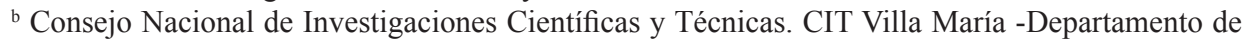
Enseñanza de la Ciencia y la Tecnología de la Facultad de Ciencias Exactas, Físicas y Naturales. Universidad Nacional de Córdoba.

${ }^{\mathrm{C}}$ Consejo Nacional de Investigaciones Científicas y Técnicas. Universidad Nacional de Río Cuarto.

* Correspondencia: Universidad Nacional de Villa María. Instituto Académico Pedagógico de Ciencias Sociales. Avenida Arturo Jauretche, 1555 - X5900 Villa María, Córdoba. Argentina.

E-mail: agumanavella@hotmail.com 
Abstract: The paper aims to advance in the study of apprenticeship in different contexts -not formal and informal-; recognizing the importance that certain personal and contextual aspects acquire in job training. A multiple case study was carried out, considering two cases: the Clavito case, a shoe repair training workshop, who learned his trade in informal contexts, and the Álvaro case, participant in the training. The results obtained allow us to recognize the interaction between personal and contextual aspects, which participate and are promising for the learning of trades; allowing to re-think these contexts and the importance of continuing to work on the design of strategies and ways of teaching in the spaces of job training.

Keywords: learning, trades, non-formal contexts, informal learning.

\section{INTRODUCCIÓN}

"Cuando el zapato te aprieta, la media te da calor" (Clavito)

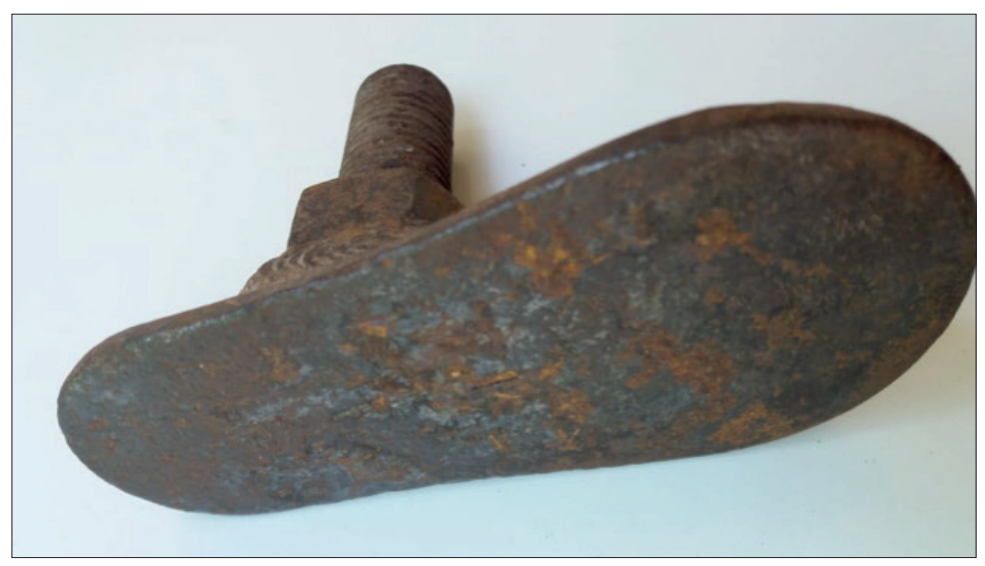

Fuente: Danilo Donolo, soporte de zapato, $2018^{1}$.

Las experiencias ajenas y a veces muy cerradas en los ámbitos personales son fuente de apreciaciones y cuasi teorías que en casos particulares desafían las imágenes que pueden aportar los big data. Los big data son muchos, pero no tienen historia, por lo que si bien dicen mucho del hoy les faltan los contextos de interpretación del pasado. Los oficios dejaron de ser lo que eran, algunos desaparecieron o están en una vía que los lleva a eso. Otros resisten

\footnotetext{
${ }^{1}$ Se reconoce actualmente este utensilio como yunque, horma de hierro para trabajos de zapatero.
} 
en algunos ámbitos reducidos ${ }^{2} \mathrm{y}$ con menor número de participantes ${ }^{3}$. Las historias sobre cómo la gente entró en un oficio o este se hizo idóneo son muchas, generalmente asociadas a la necesidad junto con la oportunidad que apareció en el momento. Mi padre, Guido, ponía media suela a los zapatos que sus pilluelos hijos destrozaban semana tras semana, jugando al fútbol en un patio de cemento o metiéndolos en cuanto charco o pantano ocasionaba la lluvia en la calle de tierra.

"Trae esos zapatos", requería. Los lavaba con cuidado, los secaba y entonces el deterioro se hacía evidente. A veces con dos o tres clavitos del $10 \mathrm{o}$ del $12^{4}$ bastaba, o alguna tachuela bien remachada permitía usarlos unos días más. En otras ocasiones el desperfecto impuesto era mayor... y entonces el reemplazo de la media suela se imponía.

Tomaba la plancha de suela de vacuno, ponía el zapatito y marcaba el contorno, tratando de desperdiciar lo mínimo y controlando que el espesor de la suela fuera similar para ambos. Recortaba por la marca, con una cuchilla especial, muy afilada y que guardaba cuidadosamente en lugares secretos del galponcito del fondo ${ }^{5}$. Después de ver el trabajo terminado y de presentar en cada zapato cada suela los metía en agua durante 24 horas (al día siguiente retomaba el trabajo); mientras tanto sacaba con cuidado los clavitos que aún mantenían la parte de arriba del zapato (luego supe que era la capellada) de la parte que pisaba, le cuidaba el detalle y limpiaba nuevamente los lugares en que iba a asentar la nueva media suela.

La historia seguía al día siguiente. Tomaba las suelas recortadas ya remojadas y las sometía a una golpiza para que tomaran más resistencia, rebajaba la parte del cuarto o la bóveda ${ }^{6}$, y nueva presentación para ver que todo estuviera en su sitio. “¿Y dónde está la horma?”, preguntaba. La horma es

${ }^{2}<$ https://es.wikipedia.org/wiki/Herrero $>$.

${ }^{3}$ Algunos se van jubilando y otros son superados por las nuevas tecnologías que abandonan el simple "lo atamos con alambre".

${ }^{4}$ iQué paradoja! Un artículo sobre zapateros tiene notas al pie y no al zapato. Del 10 o del 12 se refieren al largo del clavo en milímetros.

${ }^{5}$ De todos modos, nada impedía que entre las travesuras incluyéramos buscar ese instrumento cortador, para actividades de menor cuantía en un uso inapropiado que terminaba haciendo mella (dientecitos) en su delicado filo. La misma pregunta terminaba con un "Yo no fui, ni siquiera sé dónde está". El viejo sacudía la cabeza y marchaba por un nuevo afilado. Y la historia volvía a repetirse.

${ }^{6}$ No sabía que se llamaba así hasta que lo busqué en Google. Es a la altura en que el pie hace el arco. 
esa formita de zapato pero de hierro ubicada en un soporte de madera donde ponía el zapato por fuera y, según fuera el grosor de la suela, intentaba con un clavito afirmar la capellada a esta ${ }^{7}$. No era fácil el detalle de hacer concordar todo de manera armónica. Después del primer clavito cada cosa caía en su sitio y la media suela quedaba fijada. Tomaba un rato más remachar los clavos y poner algunas tachuelitas para que la parte media de la suela quedara fija. Y bien, consideración de lo realizado y a otro zapato ${ }^{8}$. Una buena lustrada, brillo adicional y mirada final de aprobación.

Un día de prueba en piecitos de niños y certificado de calidad aprobado. A la semana siguiente empezaba la misma historia: los destrozos eran más que evidentes.

Lo más interesante del asunto es que el viejo no era zapatero, era mecánico, aunque hacía el trabajo con la misma precisión que si armara un motor. Cuidados extremos y dedicación.

Y eso era lo más llamativo, creo, la decisión de hacer algo bien, lo mejor posible. Él sabía perfectamente que a poco de andar -literalmente hablando- los pequeños deditos de niño aparecerían por la punta de un zapato ya desahuciado y la historia se repetía.

En los últimos años, la oferta de capacitaciones en oficios ha aumentado de manera considerable. En parte, de ello se desprende el interés de realizar este escrito, que tiene por finalidad avanzar en el estudio del aprendizaje de oficios, en diversos contextos -no formales e informales-; reconociendo la importancia y el valor que adquieren ciertos aspectos personales y contextuales, implicados en los aprendizajes para el trabajo.

El trabajo se estructura en cuatro apartados. En el primero, se presentan consideraciones teóricas sobre los principales aspectos abordados en el estudio: aprendizaje situado, aprendizaje profesional y contextos educativos no formales e informales. En el segundo apartado, se mencionan los aspectos metodológicos. En el tercero, se comentan los resultados obtenidos, en relación con: trayectorias de aprendizaje en oficios, relación maestro-aprendiz,

\footnotetext{
${ }^{7} \mathrm{Si}$ bien la goma ya se había inventado, no existían como ahora las que configuran la parte inferior de zapatos y zapatillas, había que hacerlas y clavarlas. El Poxiran (marca registrada) no apareció hasta mucho tiempo después.

${ }^{8}$ Creo que el viejo habrá pensado más de una vez lo bueno de no ser una especie como el ciempiés.
} 
tareas y desafíos en el oficio de zapatero, compromiso y creatividad en el aprendizaje. Finalmente, en el cuarto apartado se exponen algunas reflexiones sobre determinados aspectos propios de los contextos no formales e informales, que resultan promisorios para el aprendizaje de oficios.

\section{Algunas CONSIDERACIONES teÓRICAS}

En cuanto al aprendizaje de oficios, son variados los constructos que nos permiten entender este concepto. La teoría del aprendizaje situado con su impronta en los oficios, el concepto de cognitive apprenticeship ${ }^{9}$ y los contextos de aprendizaje no formales e informales.

\subsection{Teoría del aprendizaje situado}

En sucesivos trabajos, Jean Lave y Etienne Wenger (Lave y Wenger, 1991; Lave, 1997; Lave, 2002; Wenger, 2001;Wenger, 2010b) empiezan a trabajar en una teoría situada del aprendizaje, que encuentra sus primeros desarrollos en la antropología social (Martín, 2016).

Algunos de los estudios etnográficos sobre el aprendizaje que realizaron Lave y Wenger (1991) enfatizaban el carácter indivisible del aprendizaje y las prácticas laborales, con el análisis de investigaciones referidas al proceso de aprendizaje y participación de las parteras mayas de Yucatán en México, los sastres de Vai y Gola en Liberia, el trabajo de los navegantes de la armada de Estados Unidos, los alcohólicos no bebedores de Alcohólicos Anónimos y los carniceros de supermercados de Estados Unidos. Estos autores piensan que las raíces de todo sistema complejo de trabajo y aprendizaje están en cada caso en la actividad laboral, en la tecnología, las carreras y las relaciones

\footnotetext{
${ }^{9}$ Collins y Kapur (2014) utilizan este término - que traducido al español significa aprendizaje cognitivo- para referirse a la modalidad bajo la cual se inician los novatos en una determinada práctica mediante la ayuda de expertos. En el presente artículo, remitiremos a dicho término como sinónimo de aprendizaje profesional.
} 
entre los novatos y expertos y entre compañeros de trabajo y profesionales (Lave y Wenger, 1991).

El concepto de aprendizaje situado se refiere al proceso de coparticipación social, que enfatiza el tipo de compromisos sociales que proporcionan el contexto adecuado para que el aprendizaje tenga lugar (Lave y Wenger, 1991). Un punto que nos interesa de esta teoría reside en la consideración del aprendizaje entendido como participación social, implicando un rol altamente interactivo y productivo por parte de los participantes, es decir, un proceso donde el conocimiento es construido socialmente más que individualmente (Martín, 2016).

Desde esta teoría se considera que parte del aprendizaje en las comunidades o grupos se desarrolla mediante un proceso de participación periférica legítima -PPL- (Lave y Wenger, 1991). Dicho concepto remite a una manera de entender al aprendizaje en contexto. La participación hace referencia a la diversidad de relaciones involucradas en las diferentes formas de pertenencia en una comunidad, consistentes en una visión relacional de la persona, sus acciones y el mundo (Lave y Wenger, 1991). La periferia se vincula al hecho de generar una posibilidad de exposición a una práctica real, "sugiriendo una apertura, un modo de ir ganando acceso, siendo variadas y múltiples las formas comprometidas e inclusivas de ser localizados en el campo de participación definido por una comunidad" (Martín, 2016, p. 208). La legitimidad se adquiere para que los participantes sean tratados como miembros genuinos, que van adoptando de manera progresiva y situada diferentes formas de práctica (Wenger, 2001). El constructo de participación periférica legítima provee una forma de expresar las relaciones entre los recién llegados, los novatos, y los veteranos, miembros expertos de una comunidad, acerca de sus actividades, conocimientos, identidades y prácticas (Lave y Wenger, 1991).

\subsection{Aprendizaje profesional}

Diversos autores (Collins, Brown y Newman, 1989; Collins y Kapur, 2014) describen la manera en que tradicionalmente se han transmitido conocimientos requeridos para desarrollar prácticas expertas, en determinados rubros de trabajo. Los autores enfatizan que tanto el aprendizaje de oficios como mu- 
chas de las habilidades complejas que se utilizan en interacciones diarias han sido incorporadas a contextos que exceden a la educación formal, mediante una enseñanza basada en la observación, el entrenamiento y la aproximación sucesiva a tareas variadas y de creciente complejidad.

Collins y Kapur (2014) enfatizan que el aprendizaje de oficios se caracteriza por una particular interacción entre maestro y aprendiz, en la que el experto brinda atención cercana, personalizada e inmediata al novato. La adquisición de habilidades propias del oficio se logra mediante una secuencia de actividades, por las que la observación repetida del experto desarrollando su labor, el entrenamiento del aprendiz con los andamios necesarios y la práctica de tareas resultan fundamentales.

Los autores avanzaron en el estudio del aprendizaje de oficios, distinguiendo las particularidades de dos tipos de aprendizajes: el aprendizaje tradicional y el cognitive apprenticeship. Respecto a este último concepto, corresponde mencionar que haremos referencia al término aprendizaje profesional para hacer alusión a dicha noción. De esta forma, los autores destacan que el aprendizaje tradicional surge mediante la colaboración del novato en diversos trabajos, propios de un oficio, realizados por expertos. Este tipo de aprendizaje emerge en el contexto donde acontecen las demandas y, por ende, se ve condicionado por las labores solicitadas. Mientras que el aprendizaje profesional emerge en diversos contextos. En este último se persigue un objetivo primordialmente pedagógico, razón por la cual los expertos eligen las tareas y problemas que deben ser trabajados, así como las técnicas que deben enseñarse y las habilidades que se han de transmitir, plausibles de ser aplicadas en una variedad de situaciones.

\subsection{Contextos de aprendizaje no formales e informales}

Trilla, Gros, López y Martín (2003) realizan una crítica a la exclusividad con la que suele ser vinculada la función educativa a las instituciones escolares. Los autores remiten a diversos cambios que se fueron gestando en la sociedad -tanto en la vida familiar como en el mundo del trabajo- y que introdujeron la demanda de una educación permanente, la cual debe ser abordada desde contextos que trascienden el sistema de escolarización reglado. 
Es aquí donde cobran importancia los contextos no formales e informales para la formación en el trabajo. En este sentido, los autores consideran que una de las funciones principales que tienen los contextos no formales de aprendizaje está relacionada con el trabajo, específicamente con la formación laboral, considerándolos como los principales ámbitos orientados al mundo laboral, mencionando la necesidad de replantear las relaciones entre el mundo del trabajo y el de la educación. Dentro de las particularidades de los contextos no formales los autores señalan que los objetivos suelen ser específicos, sectoriales y delimitados, orientados a producir efectos a corto plazo; la participación se origina a partir de intereses y necesidades personales y conscientemente asumidas, siendo muy variable la formación previa que presentan los docentes; se tienden a considerar las necesidades autóctonas e inmediatas para seleccionar los contenidos más idóneos. La mayor parte de los programas no formales se realizan en tiempo parcial y tienen una duración menor que la de los cursos académicos; los horarios son flexibles; los contenidos abordados son poco teóricos y abstractos; se valora la experiencia práctica de los sujetos y sus conocimientos informalmente adquiridos.

Por su parte, el aprendizaje informal es aquel que resulta de las actividades diarias relacionadas principalmente con el trabajo, la familia y el ocio (Colardyn y Bjornavold, 2004). La organización del aprendizaje informal tiene algunas características compartidas en muchos entornos: no es táctica; está incrustada en la actividad significativa; se basa en la iniciativa, el interés o elección del aprendiz, y no implica una evaluación de la actividad.

\section{Aspectos metodológicos}

La investigación cualitativa se fue estableciendo gradualmente en el campo de las ciencias sociales y la psicología, teniendo relevancia específica para el estudio de las relaciones sociales (Flick, 2004). En el campo de la psicología educacional y desde la teoría situada, abordar los estudios cualitativamente implicaría centrarse en los procesos de coparticipación y en las relaciones que giran en torno al aprendizaje.

Los métodos cualitativos permiten recoger datos descriptivos, considerando las palabras y conductas de las personas implicadas en la investigación y 
permitiendo la realización de un estudio fenomenológico de la vida social (Taylor y Bogdan, 1986).

Con la intención de comprender en profundidad cómo se desarrolla el aprendizaje de oficios en diversos contextos, se realizó un estudio de caso múltiple (Stake, 1998). En palabras de Simons (2011), el "estudio de caso cualitativo valora las múltiples perspectivas de los interesados, la observación en circunstancias que se producen de forma natural y la interpretación en contexto" (p. 21), atendiendo a las formas en que los participantes construyen e interpretan sus mundos.

En el presente estudio, para conocer más sobre los contextos donde se desarrolla el aprendizaje de oficios, se consideraron dos casos: por un lado, el caso Clavito, tallerista de una capacitación, quien aprendió su oficio en contextos informales, y por otro lado el caso Álvaro, participante de la capacitación.

La capacitación fue ofrecida por la municipalidad de Villa María (provincia de Córdoba, Argentina) y se llevó a cabo de junio a noviembre, en el año 2016. A esta asistían semanalmente siete personas a aprender el oficio de zapatero.

Atendiendo a las trayectorias y a la dinámica de las interacciones generadas en el aprendizaje del oficio, realizamos entrevistas individuales y semiestructuradas, con una duración aproximada de sesenta minutos cada una.

\section{LOS DATOS Y SUS ANÁLISIS}

A partir del análisis de las entrevistas y teniendo en cuenta los planteamientos teóricos mencionados, consideramos cuatro dimensiones que hay que tener en cuenta para el aprendizaje de oficio:

\subsection{Trayectorias de aprendizaje en oficios}

Mediante las entrevistas realizadas al participante de la capacitación y al tallerista, hemos podido reconocer las singularidades en sus trayectorias de formación, a nivel general y particularmente, en relación con el aprendizaje 
de oficios; sus primeros trabajos, la forma de ir aprendiendo y adquiriendo dominio y experiencia en el campo.

En el caso de Álvaro, su formación y experiencia en contextos educativos formales fue haber realizado sus estudios primarios, hasta séptimo grado. De adulto comenzó a transitar contextos no formales de capacitación para el trabajo. Primero realizó un curso sobre soldadura, oficio en el que no llegó a desempeñarse. Luego, en el año 2016, comenzó la capacitación de reparación de calzado, y los primeros aprendizajes en torno a este trabajo fueron en contextos informales, mediante conversaciones con un vecino de su pueblo que anteriormente ejercía el oficio.

Vine por una necesidad que había en casa. Mi sueldo es poco y un día surgió que había que arreglar un zapato para mi hija, que es abanderada y tenía un acto. Me fui hasta lo de un muchacho que vivía de esto y me dice: No trabajo más porque no me gusta". Me explicó cómo hacerlo, yo lo hice y se lo llevé a él para que lo viera. Me dice: "Está perfecto". Al otro día me mandó clientes a mi casa.

El fragmento de entrevista citado da cuenta de cómo fueron los comienzos de Álvaro en el oficio y de los motivos que lo impulsaron a querer aprender a reparar calzados. La necesidad de que uno de sus familiares necesitara arreglar sus zapatos, sumado a que quien desempeñaba el oficio de zapatero en el pueblo ya no ejercía su labor y a las necesidades económicas por las que se encontraba atravesando su familia, posibilitó que Álvaro decidiera comenzar a aprender el oficio, primero reparando aquel par de zapatos que su hija necesitaba en carácter de urgencia, luego tomando trabajos de vecinos y conocidos de su localidad, y finalmente capacitándose en el curso de reparación de calzado, ofrecido por Clavito.

En el caso del tallerista, su experiencia en contextos formales ha sido dificultada por diversas circunstancias personales y familiares, lo que llevó a Clavito a realizar sus estudios primarios en varias escuelas -dependiendo del lugar de trabajo de su madre-. En paralelo al cursado de sus estudios primarios, Clavito comenzó a aprender el oficio de zapatero, realizando algunos trabajos en el rubro: 
(...) Cuando tenía 9 años, éramos cinco de familia, mi papá tenía un solo sueldo y me compraba las alpargatas una sola vez al mes, para poder ir al colegio, a veces llegaba a los quince días y estaba ya con los dedos afuera. Entonces mi tío que era zapatero me rescató, me enseñó a lustrar zapatos. Él me los ponía en una caja, yo los terminaba de lustrar en la máquina y se los entregaba a los clientes y ahí me fui ganando la propina. Con eso me compraba las zapatillas y los bizcochos (...).

Tras recibir una beca para realizar la secundaria, Clavito decide postergar sus estudios para poder trabajar y ayudar a su familia. Retomó su educación secundaria años después, en un colegio nocturno para adultos.

Las dificultades económicas por las que pasaba su familia fueron el principal motivo por el que Clavito se inició en el oficio de la reparación de calzados. Sus aprendizajes en el rubro fueron adquiridos en contextos educativos informales. Sus primeros acercamientos con el oficio fueron de niño, mediante la observación del oficio y pasar tiempo con su tío, que oficiaba de zapatero.

A sus 17 años comenzó a trabajar en una fábrica de creación de calzado, oficiando en sus inicios de cortador de zapatos y luego de ayudante del modelista, para quien creaba escalas de moldes de calzado. Paralelamente a dicho trabajo Clavito comenzó a ejercer el oficio en su hogar: empezó cortando moldes para fábricas de mocasines, hasta que finalmente decidió abrir su propio taller de calzado, con la ayuda de su esposa, a quien le enseñó a coser zapatos. Juntos desempeñaban el oficio.

\subsection{Relación maestro-aprendiz}

En este apartado se pretende hacer hincapié en el vínculo que se generó entre el tallerista y el participante durante la capacitación en el oficio de reparación de calzado.

En el caso de Álvaro, la capacitación fue su primer acercamiento al oficio, por lo que la relación que estableció con el tallerista resultó fundamental para iniciarse en el aprendizaje y en el rubro de la reparación de calzado: 
(...) Qué decir del profesor. Es una persona abierta, que no se guarda los secretos, porque todo nos ha ido enseñando. Es dejar un legado de lo que está haciendo él en nosotros, a mí me está enseñando el oficio (...) abrirte las puertas de su casa, poner las máquinas a nuestra disposición para que uno quedara bien con un trabajo (...) con Clavito tenemos una relación muy buena, atiende a uno, atiende al otro, nadie se queda con dudas, con ganas de preguntar.

Este fragmento permite reconocer la relación experto-novato que se generó entre Clavito y Álvaro. Del mismo modo que se trabajaba en la capacitación, el tallerista ofrecía su taller - que se encuentra en su casa-, poniendo a disposición de los participantes sus herramientas y maquinarias, en horarios extras a los de la capacitación.

El participante no solo tuvo un vínculo significativo con el tallerista, sino también con sus compañeros de capacitación, lo que generó un marco para el aprendizaje colaborativo:

(...) el compañerismo que tenemos, nos escribimos siempre (...) hacerlo en casa está bien, pero te falta el haber compartido con todos (...) trataremos de seguir en contacto con el grupo, con el profesor, para ver cómo les va a otros. Yo creo que E. es especialista en cambiar cierres y yo no sé si me animaría a cambiar un cierre (...) entonces por ahí es bueno saber que hay alguien acá que lo puede hacer. Es como un cambio: yo le doy para que ella me cambie el cierre y ella me pide que le haga el taco. Eso nos va a mantener siempre en contacto, no vamos a perder la esencia de este grupo.

La relación que se generó entre los compañeros también trascendió el marco de la capacitación. Un espacio construido conjuntamente y generado más allá del taller, por los participantes y el tallerista. En un grupo de WhatsApp escribían diariamente tanto sobre cuestiones relativas al aprendizaje del oficio como para pedir ayuda por una demanda de trabajo, asistir al taller del profesor para pedir herramientas y consejos ante un desafío laboral, o acerca de otras cuestiones, como el envío de chistes, buscando así conversar o incluso organizar reuniones y de ese modo reencontrarse.

Álvaro había participado con anterioridad en otro contexto no formal de formación para el trabajo, específicamente en un curso de soldadura. En el fragmento que compartimos a continuación se expone una comparación que 
él realiza entre las dos capacitaciones, señalando la estructura del curso y la relación con el profesor.

Hace tres años hice un taller de soldadura, en la UTN. Pero quedó todo ahí nomás, no lo llegué a disfrutar porque éramos muchos y las máquinas para realizar la tarea eran poquitas (...) No se dio nada como para decir que yo pudiera haber desempeñado ese conocimiento en ningún lado (...) yo ya tengo la experiencia de otro curso. La gente muy buena, pero nunca más nos vimos; con el profesor prácticamente no dialogábamos. No como con Clavito.

Para Álvaro el vínculo que se formó entre los compañeros de la capacitación y el tallerista, en el marco de la capacitación en el oficio de reparación de calzado, resultó motivador para el aprendizaje, y destaca que entre quien enseña -y conoce el oficio-y los participantes - nuevos en el rubro de la zapatería- se había generado una relación cercana, lo que posibilitó el diálogo, el aprendizaje, compartir -ya fuera experiencias, conocimientos o herramientas- y momentos de disfrute.

En el caso de Clavito, que ejerce el oficio de zapatero desde hace más de cuarenta años, ha tenido diversas y variadas experiencias en lo referente a la relación maestro-aprendiz durante su trayectoria en el rubro de la zapatería:

(...) Un jefe que había en la fábrica fue mi maestro. Era un señor que, cuando vos hacías un mal trabajo, él no te retaba, venía y te explicaba y te enseñaba cómo hacerlo. Entonces yo me rescaté y él me llevó a la mano guía.

No es casual que a la persona que más le enseñó sobre el oficio de compostura de calzado Clavito lo haya considere "su maestro". La relación que ha tenido con este señor y el modo con la que le enseñó desde muy temprano el oficio han resultado significativas para él y dicho vínculo es similar al tipo de relación que Clavito mantiene con sus aprendices para enseñar el oficio.

(...) Los viejos zapateros eran muy egoístas, no te enseñaban y yo, como tengo vocación de servicio, no soy egoísta, tengo la virtud de poderles enseñar todos los secretos (...) yo les digo que pregunten, que no tengan miedo, yo no tengo ningún problema en que vayan a mi casa para poder explicarles, yo estoy pendiente de corregirlos en el momento. 
Las citas recuperadas de la entrevista con Clavito dan cuenta de la relación que mantiene con quienes se encuentran aprendiendo el oficio y de cómo él, como tallerista y experto en el rubro, hace lo posible para explicar y transmitir a los novatos zapateros todo lo que conoce: contarles los "secretos del oficio", ofrecerles su taller para que se acerquen a preguntarle sobre demandas de trabajo, brindarles herramientas e insumos para que puedan realizar las labores, generando de ese modo confianza para que los aprendices se sientan seguros $\mathrm{y}$ realicen preguntas, hagan comentarios o pidan explicaciones.

\subsection{Tareas y desafios en el oficio de zapatero}

En este apartado detallaremos los diversos trabajos y desafíos a los que se han enfrentado Álvaro y Clavito en sus trayectorias laborales relacionadas con el oficio de zapatero.

En el caso de Álvaro, el primer desafío con el que se encontró fue antes de comenzar la capacitación: al enterarse de que el único zapatero del pueblo ya no ejercía su labor decidió reparar él mismo los zapatos que su hija necesitaba. Luego, en el marco de la capacitación, se enfrentó a diversas prácticas, propias del oficio:

(...) Modifiqué zapatos que ya vienen de fábrica y traté de amoldarlos a gusto del cliente (...) en este caso, la señora no puede pisar con la parte delantera del pie porque le hace mucho daño y lo había dejado de usar por eso. Entonces, ¿qué me dice el profesor?, que hay que rellenar en la puntera de adelante, se puede bajar la parte del talón, eso va a hacer que el zapato se incline hacia atrás. (...) le arreglé un par de botas a una mujer que necesitaba cambiarle los tacos para más comodidad. Ella es una persona grande, pesada, y eran tacos aguja. El profesor me dijo: "Se le puede cambiar el taco, dándole mayor comodidad, con un taco de la misma altura, pero más ancho" (...) aprendí a conocer la calidad de los cueros, o de qué material está hecho el zapato.

Los fragmentos de entrevistas recuperados permiten comprobar que las diversas tareas que Álvaro fue aprendiendo y realizando durante la capacitación corresponden a labores que le fueron demandadas por personas conocidas, enteradas de sus comienzos en el oficio. En todas las situaciones descritas por 
Álvaro se reconoce la ayuda del tallerista, en la búsqueda de opciones para resolver los problemas planteados por los clientes y en la descripción y explicación de los pasos que hay que seguir para realizar el trabajo.

En los fragmentos que recuperamos a continuación podemos apreciar que enfrentarse a diversas tareas propias del oficio de zapatero implica para Álvaro el aprendizaje de dicha labor, la posibilidad de buscar y crear soluciones y alternativas frente a las demandas de sus clientes y la alegría de verlos contentos con el trabajo realizado.

Son cosas que voy poniendo en práctica. Yo lo estoy aprendiendo con la práctica del taller y yendo aparte al taller del profesor (...) la clienta quedó tan conforme que me hizo propaganda por todo el pueblo (...) es algo que a mí me da una gran satisfacción, porque me veo como un creador. Porque hay situaciones que se presentan, que solamente la astucia y la inteligencia, para poder resolver un problema, a lo mejor de un zapato.

En el caso del tallerista, sus desafíos en la práctica del oficio guardan relación con cuestiones que resultaban novedosas para él, pues apenas se iniciaba en el rubro. Son situaciones que ha logrado superar y de las que ha aprendido cosas que desconocía de la zapatería y que le han servido para poder asumir trabajos que le demandaban:

Los primeros trabajos fueron hacer taco y medias suelas de goma y con el tiempo empecé a probar a hacer la media suela cosida, pero no tenía quién me cosiera, entonces tuve que aprender. "Cuando el zapato te aprieta, la media te da calor", dicen. Tuve que aprender a hacerlo por necesidad. Porque yo quedo sin trabajo y había cosas que sabía y cosas que no sabía, que había visto pero que no había practicado.

A lo largo de su trayectoria y experiencia en el oficio, se ha enfrentado a diversas demandas y ha realizado una amplia variedad de tareas relativas al oficio de zapatero:

Cuando tenía nueve años mi tío me enseñó a lustrar zapatos (...) en la fábrica me dijeron: "Nosotros te vamos a enseñar, vas a oficiar de cortador de zapatos con cuerdas de reloj". (...) el modelista me rescata y me lleva a hacer moldes junto con él, a hacer la escala de los modelos. (...) los fines de semana me 
ganaba la changa cortando zapatos mocasines (...) de noche trabajaba en la fábrica, cortando zapatos con troqueladora (...) con mi esposa trabajábamos juntos en la compostura y en la fabricación de zapatos (...) Me encargo de la compostura en general de zapatos, carteras, reformas, teñidos, tacos. En un día de trabajo tenés de todo: reformas, teñido, recortar media suela, una manija de cartera, poner cierres, reparación de zapatillas y reformas.

Las expresiones recuperadas permiten reconocer la variedad de trabajos que Clavito ha realizado durante su trayectoria en el oficio y que realiza diariamente, referidas a problemas genuinos, propios de la compostura de calzado.

Siendo ya un reconocido reparador de calzados en la ciudad, los trabajos con los que se enfrenta actualmente tienen que ver con tareas novedosas y especiales, trabajos que le demandan sus clientes para los que debe encontrar soluciones, como en el caso de personas con ciertos problemas físicos, para que puedan caminar y utilizar diversos calzados sin sufrir molestias o dolores:

(...) Hago trabajos especiales, delicados... a personas mayores que se les quiebra la cadera y a otras personas que han sufrido accidentes... necesitan que se les haga un realce de unos centímetros en el calzado.

Actualmente -desempeñándose como zapatero y como tallerista- Clavito no solo se enfrenta a diversas tareas en su taller, sino que además genera retos en los participantes de la capacitación mediante los cuales enseña a realizar ciertos trabajos propios del oficio:

La última clase les llevé un par de zapatos de mi taller, y les dije: "Esto es para teñir". Yo me senté a hacer otro trabajo y ellos se dedicaron a teñir. Ellos ya saben que tienen que desteñirlo con virulana, que tienen que usar el alcohol, que tienen que pasarle la tinta, sacar la base de abajo, sacarles el brillo y utilizar en el teñido tinta al alcohol.

En el fragmento de entrevista recuperado se puede apreciar que los desafíos que propone Clavito a los participantes de la capacitación les permiten realizar labores directamente relacionadas con demandas de trabajo que él recibe en su taller y trabajar de manera independiente, con la menor ayuda posible por parte del tallerista, de modo que articulen e implementen en dicho trabajo todo lo aprendido durante la capacitación. 
Voy preparando de acuerdo a cómo veo que responde el grupo (...) El desafío es enseñarles cosas más complicadas. Al ver que tienen ganas, sé que las van a aprender. Los veo motivados y les enseño los secretos.

Durante la capacitación, el tallerista enseñó a los participantes a crear determinadas herramientas. En el siguiente fragmento, Álvaro relata cómo creó sus propias herramientas de trabajo:

A la multiuso la aprendimos a hacer, yo la hice con un caño de aluminio porque no tenía cable de cobre en mi casa y con cuerda de reloj, que había en un aparador en casa; es lo que estoy usando ahora para el corte de cuero, gomas o lo que sea. Después me hice una lima, porque no tenía cómo llegar a la parte de las terminaciones de la suelina (...) me pude comprar una moledora de banco, a la cual le adapté uno de los lados (...) desarmé el reloj, vi unos engranajes; así que acudí a un cabito de cepillo de dientes y me hice un fijador de puntos.

Mediante las citas recuperadas podemos reconocer que, a medida que le fueron demandando diversos trabajos, Álvaro se fue enfrentando a múltiples desafíos. El no contar con todas las herramientas que necesitaba para llevar a cabo las labores solicitadas hizo que fuera creando las suyas, tanto con materiales sugeridos por el tallerista como con elementos similares, obtenidos de diversos objetos en desuso que tenía en su hogar, así como realizando adaptaciones a herramientas que fue adquiriendo.

\subsection{Compromiso y creatividad en el aprendizaje}

Los desafíos que les propone Clavito a los aprendices guardan relación con el compromiso y la creatividad que manifiestan y desarrollan los participantes para aprender el oficio. A continuación, se recuperan fragmentos de la entrevista en la que el tallerista detalla diversas situaciones en las que los participantes de la capacitación demuestran estar comprometidos en el aprendizaje del oficio de zapatero: 
Yo noto las ganas que tienen en la asistencia y en la preocupación cuando un día no pueden ir, me llaman por teléfono y me preguntan si pueden venir a mi casa para ir aprendiendo y no perderse lo que hemos visto en el curso (...) Álvaro se aboca, viene a mi casa porque quiere seguir aprendiendo, se compromete con los trabajos que le llevan porque los quiere entregar a la perfección.

En el fragmento que citaremos a continuación, Álvaro hace referencia al compromiso compartido de aprender el oficio, haciendo mención a su desempeño durante la capacitación:

Yo escucho siempre lo que el profesor explica y nos aconseja. Eso creo que me ha ayudado a desempeñarme con confianza, por haber prestado atención.

En los primeros trabajos de reparación de calzado por parte de Álvaro, se puso en juego no solo el compromiso compartido hacia el aprendizaje del oficio, sino también su creatividad, lo que le permitió resolver diversas tareas que le fueron demandadas con las escasas herramientas que había adquirido, realizando adaptaciones de algunas y creando otras que le permitan llevar a cabo la labor. A continuación, se recupera un fragmento de entrevista en el que Álvaro reconoce la importancia de ser creativos, para ejercer el oficio:

Y bueno, la creatividad. Porque todo eso implica darse maña para poder hacer las herramientas, para poder salir adelante. No todo se puede comprar en la vida. Yo trato de realizar los trabajos con lo poco que uno va creando.

Para Clavito también resulta fundamental desarrollar la creatividad, para ejercer el oficio:

Todos los trabajos necesitan de la imaginación y la creatividad... yo a los chicos siempre les digo: "Ustedes tienen que tener imaginación". Yo les enseñé lo básico y después la imaginación te ayuda a resolver y a ver cómo hacés el trabajo que te traen (...) Qué mejor creatividad que la que le vi a Álvaro. Él se planteó cómo hacer para coser los botines. Yo por teléfono le expliqué: "Buscá una lesna". Y resulta que me sorprendió, porque buscó una varillita de limpiaparabrisas y se hizo una aguja crochet flexible. La imaginación surge cuando hay necesidad económica y las crisis actuales les obligan a crear cosas. 
En el fragmento de entrevista podemos apreciar que, para Clavito, la creatividad guarda relación con el compromiso que Álvaro construyó en el aprendizaje del oficio, con la escasez de herramientas con las que cuenta aún para desempeñar sus primeras labores en el rubro y con la necesidad de contar con esas herramientas para poder trabajar y obtener nuevos ingresos.

\section{Algunas Reflexiones}

El presente estudio nos permite reflexionar en relación con diversos aspectos que interactúan y resultan promisorios para el aprendizaje de oficios.

Las cuatro dimensiones analizadas -trayectorias de aprendizaje, relación maestro-aprendiz, problemas y desafíos en la práctica del oficio y compromiso y creatividad en el aprendizaje- muestran la articulación y el interjuego entre aspectos personales y contextuales que participan en el aprendizaje del oficio (Finn y Zimmer, 2013).

El análisis de las trayectorias de aprendizaje de Álvaro y Clavito en el oficio de la reparación de calzado permite reconocer la importancia que adquieren las relaciones e interacciones entre maestro y aprendiz, así como la participación y el trabajo en tareas y problemas genuinos relativos al oficio de zapatero. En este sentido, resultan relevantes los aportes de las teorías de Lave y Wenger (1991) y de Collins y Kapur (2014), quienes nos permiten pensar el aprendizaje de oficios en estos contextos, a partir de la construcción social de conocimientos compartida y de la práctica situada, lo que posibilita que el oficio sea aprendido mediante el trabajo con problemas reales, a partir del desarrollo de ciertas habilidades y destrezas que permitan aplicar lo aprendido a la variedad de trabajos demandados.

El análisis realizado permite reconocer que el vínculo que Álvaro (novato, aprendiz del oficio) estableció con Clavito (experto, maestro en el rubro de la zapatería), sumado a las tareas desafiantes que el tallerista propuso durante la capacitación -aspectos contextuales-, posibilitaron la construcción compartida de un compromiso por el aprendizaje y el desarrollo de la creatividad -aspectos personales-, lo que a su vez permitió que Álvaro asumiera nuevos retos, adquiriera nuevas habilidades y se enfrentara a problemas genuinos y a prácticas reales del oficio. 
Un aspecto interesante sobre el tópico estudiado es la flexibilidad característica de los contextos educativos no formales. El análisis de ambas trayectorias permite reconocer que en estos espacios de capacitación para el trabajo se prioriza la experiencia en el oficio por encima de la exigencia de títulos académicos y poder así desempeñar el trabajo de tallerista, organizándose los contenidos que deben ser trabajados en la capacitación en función de los intereses y necesidades de los participantes (Trilla et al., 2003).

Los datos obtenidos de las entrevistas realizadas, permiten repensar estos contextos de formación para el trabajo y la importancia de continuar trabajando en el diseño de estrategias y formas de enseñanza en los espacios de capacitación laboral, de manera que las propuestas de formación no queden solamente supeditadas a las experiencias de los talleristas, sino que además su experticia enriquezca programas de formación diseñados acorde a las necesidades educativas y laborales de los participantes y a las demandas de trabajo actuales. En este sentido, cobran relevancia los aportes de Petrus (1997) en relación con el concepto de pedagogía social. Para el autor, la finalidad de los contextos de capacitación laboral es educar para y en el trabajo, formando a los participantes en la actividad y en valores profesionales, promoviendo el desarrollo de habilidades y la adquisición de competencias que les permitan insertarse y desempeñarse en el ámbito de las relaciones laborales.

Y es la decisión lo que hace la diferencia en cualquier trabajo, obra o realización. "Lo voy a hacer muy bien, aunque no sea lo mío. Y soy capaz de hacerlo" es la cantinela que debe sonar en mi interior. En todo oficio, en toda gestión o faena, tiene que estar ese componente de decisión de hacerlo bien. Esta cualidad es una realidad en la mayoría de las personas. Si no cómo se explican el tesón de una madre por llevar a su niño cada mañana a la escuela ${ }^{10}$, los emprendimientos liderados por mujeres ${ }^{11}$, el planeamiento y la realización de megaconstrucciones ${ }^{12}$ y un largo etcétera de ejemplos. El componente adicional a la decisión es la esperanza y la ilusión que puedan ponerse a la hora

\footnotetext{
$10<$ https://www.clarin.com/viva/julieta-lanteri-pionera-sentidos_0_RxutaMV_5.html\#cxrecs_s $>$ Lanteri toda una pionera.

$11<$ https://translate.google.com/translate?hl=es\&sl=en\&u=https://www.statmodel.com/examples $/ \&$ prev $=$ search $>$.

$12<$ https://www.youtube.com/watch?v=yM52YOSDpWQ>. Obra para Buenos Aires 2060.
} 
de imaginar y llevar a cabo un proyecto; eso es lo que nos da la energía para persistir sin tomar los caminos del desgano, del fastidio o de la inacción ${ }^{13}$.

Los oficios y los requerimientos para atender empleos irán cambiando, pero la actitud general de realización seguirá teniendo el mismo ímpetu que la mostrada por los romanos para hacer un imperio o la sociedad moderna para llegar a la luna.

Ahora, ¿usted de qué lado está? ${ }^{14}$

\section{BibliografíA}

Colardyn, D. y Buornavold, J. (2004). Validation of Formal, non-Formal and Informal Learning: policy and practices in EU Member States. European Journal of Education 39(1), 11-25. Recuperado de: $<$ https://www.competences.info/ibak/root/img/pool/docs/open/bjornalvold_colardyn_example_en.pdf $>$.

Collins, A. y Kapur, M. (2014). Cognitive apprenticeship, en R. K. Sawyer (ed.), The Cambridge Handbook of the Learning Sciences (pp. 109-127). Cambridge, UK: Cambridge University Press.

Collins, A., Brown, J. S. y Newman, S. E. (1989). Cognitive apprenticeship: Teaching the crafts of reading, writing, and mathematics, en L. B. Resnick (ed.), Knowing, learning, and instruction: Essays in honor of Robert Glaser (pp. 453-494). Hillsdale, NJ, US: Lawrence Erlbaum Associates, Inc.

FinN, J. y Zimmer, K. (2013). Student engagement: what is it? Why does it matter?, en S. L. Christenson, A. L. Reschly y C. Wylie (ed.), Handbook of Research on Student Engagement (pp. 97-131). Nueva York: Springer. FLICK, U. (2004): Introducción a la investigación cualitativa. Madrid: Morata.

$13<$ https://www.lanacion.com.ar/opinion/columnistas/sin-titulo-nid2224355>. "Solo nos queda la esperanza (...) un muy sentido mensaje ante la adversidad (...) retrato de los que solo les queda hacer frente a la adversidad". Santiago Kovadloff.

14 "Yo no pude iniciarme en el oficio de zapatero remendón. Me ganó el tiempo con la aparición de nuevos materiales que hicieron de la media suela un artículo obsoleto, dando paso a las suelas inyectadas. Sin embargo, los zapatos con suela de cuero siguen existiendo. Mirá, hay marcas y modelos de zapatos que me resultan hasta impronunciables: Louboutin, Blahnik, Choo, Aquazzurra, Alden Corthay, Santorini (seguro que en esas empresas no me van a contratar siquiera, sobre todo por la edad)". 
LAVE, J. (1997). The culture of acquisition and the practice of understanding, en D. Kirshner y J. Whitson (eds.), Situated Cognition. Social, semiotic, and psychological perspectives (pp. 17-35). Mahwah, NJ: Lawrence Erlbaum Associates.

LAVE, J. (2002). La práctica del aprendizaje, en S. Chaiklin y J. Lave (comps.), Estudiar las prácticas. Perspectivas sobre actividad y contexto (pp. 1545). Buenos Aires: Amorrortu.

Lave, J. y Wenger, E. (1991). Situated Learning: Legitimate Peripheral Participation. Cambridge, UK: Cambridge University Press.

Martín, R. B. (2016). Los procesos de participación periférica legítima en dos contextos diversos. Revista Fronteiras 3, 5. Recuperado de: $<$ http:// revistas.unievangelica.com.br/index.php/fronteiras/article/view/2050>.

Petrus, A. (coord.) (1997). Pedagogía Social. Barcelona: Ariel Educación.

Simons, H. (2011). El estudio de caso: Teoría y práctica. Madrid: Morata.

Stake, J. (1998). Investigación con estudios de caso. Madrid: Morata.

TAYLoR, S. J. y Bogdan, R. (1986). Introducción a los métodos cualitativos de investigación. México: Paidós.

Trilla, J., Gros, B., López, F. y Martín, M. J. (2003). La Educación fuera de la Escuela: Ámbitos No Formales y Educación Social. Barcelona: Ariel Edición.

Wenger, E. (2001). Comunidades de práctica. Aprendizaje, significado e identidad. Barcelona: Paidós Ibérica, S. A.

Wenger, E. (2010b). Communities of practice and social learning systems: the career of a concept. Chapter 11, en C. Blackmore (ed.), Social Learning System and communities of practices (pp. 179-198). Londres: Springer. 